\title{
Peritoneal flap mesh hernioplasty: A reliable option for difficulty in closure of complex ventral hernia: A case report
}

Bhawani Khanal*iD, Sunit Agrawal, Suresh Sah, Roshan Gurung, Rakesh Kumar Gupta

Department of General Surgery and MIS Unit, BP Koirala Institute of Health Sciences, Dharan, Nepal

\begin{abstract}
Peritoneal flap mesh hernioplasty is a technique to deal with complex ventral hernias in which primary closure of the defect is not attainable. We are here presenting a case report of a 50-year-old gentleman with huge complex ventral hernia with loss of domain. The patient also had overlying ulcerated and blackish colored skin. Botulinum toxin was infiltrated into the muscles of anterior abdominal wall and was followed by peritoneal flap mesh hernioplasty after one month. The final outcome was complete closure of the midline with a healthy skin with no evidence of abdominal compartment syndrome. There was no evidence of early recurrence on follow up.

Owing to the favorable outcome in this case, suitability of peritoneal flap mesh hernioplasty technique in treatment of complex ventral hernia could be explored further where closure of the primary defect is difficult.
\end{abstract}

Keywords: Complex ventral hernias; Component separation techniques; Peritoneal flaps

\author{
Correspondence to: \\ Dr. Bhawani Khanal \\ Assistant Professor, General Surgery \\ General Surgery and MIS Unit, BPKIHS, Dharan, \\ Nepal \\ E-mail: itsmebhawanikhanal@gmail.com
}

Submitted: August 21, 2020

Accepted: October 16, 2020

To cite: Khanal B, Agrawal S, Sah S, Gurung R, Gupta RK. Peritoneal flap mesh hernioplasty: A reliable option for difficulty in closure of complex ventral hernia: A case report.

JGMC Nepal. 2020;13(2):188-91.

DOI: 10.3126/jgmcn.v13i2.30699

\section{INTRODUCTION}

European society of hernia has defined any hernia as complex hernia which meets the following criteria. 1) Hernia with width $>10 \mathrm{~cm}$, Loss of domain of $>20 \%$, 2) contaminations and soft tissue condition, 3) Recurrent hernia with use of mesh previously, comorbidities, history of abdominal dehiscence 4) clinical scenarios such as emergency operation with bowel resection. ${ }^{1}$

Complex ventral hernia repair has been a challenging task because of the difficulty in primary closure of the defects. Peritoneal flap mesh hernioplasty is one of the techniques to deal with such problem. It utilizes redundant tissue from the hernia sac to bridge the fascial gap, and it increases the abdominal domain by expanding the abdominal wall at the site of herniation without inducing weakness laterally which is one of the flaws present in component separation techniques (CSTs). ${ }^{2}$ It can also be used for certain wide defects especially those arising from non-midline incision where component separation technique is not applicable. ${ }^{3}$

In most of the cases of long standing complex ventral hernias, loss of domain is found. It refers to the loss of abdominal viscera right to 'belong' inside the abdominal cavity. Infiltration of abdominal muscles preoperatively with Botulinum toxin bilaterally and progressive preoperative pneumoperitoneum (PPP) are the techniques to deal with. Botulinum toxin lengthens 
and relaxes the laterally retracted abdominal muscles and enables easy closure of large complex ventral hernias. ${ }^{4}$

Assessment of increase in domain is done by preoperative non contrast computed tomography (NCCT) of abdomen and pelvis before adopting such techniques and repeating the same at the time of surgery. NCCT of abdomen and pelvis also helps us to rule out chronic abdominal abscess and malignancy that are sometimes confusing with chronic long standing ventral hernia.

In this case report, we aim to describe the use of transposed flaps of preserved sac which effectively extend the fascial layers to support and envelop the mesh, sandwiching it between two layers of autologous tissue in a relatively tension free manner.

\section{CASE REPORT}

A 50-year-old gentleman presented with huge midline anterior abdominal wall swelling involving the epigastric, umbilical and hypogastric region for 4 years duration. He had undergone exploratory laparotomy and Graham's omental patch repair for duodenal ulcer perforation 8 years back. The swelling was gradually progressive in size and used to increase while doing strenuous exercise. Initially the swelling was completely reducible on its own, but for last one year it became incompletely reducible. On examination, there was a huge bulge in abdomen with blackish discoloration of overlying skin along with ulcerations at places (Fig 1a, 1b). On palpation, cough impulse was positive, herniated bowel loops as well as widely separated sheath could be felt. Content of the hernia was not completely reducible. As this was the long standing ventral hernia NCCT was done, which revealed the size of fascial defect around $21 \mathrm{~cm}$.

Abdominal muscles were infiltrated bilaterally with Botulinum toxin 150 IU. On reassessment after 1 month, size of fascial defect reduced to around $15 \mathrm{~cm}$ on NCCT. The patient was then planned for peritoneal flap mesh hernioplasty.

Firstly, a vertical midline incision to the skin was given and abdomen was opened. There was mild adhesion for which adhesiolysis was done. Skin flaps were raised bilaterally preserving the sac of the hernia. Flaps were raised till the sheath was reached bilaterally (Fig 2a). Subsequently, retrorectus space on right side was entered after incising the anterior sheath. Similarly, retrorectus space on left side was entered after incising the posterior sheath. Peritoneal flap was then sutured with posterior sheath of right side to the posterior sheath on left side (Fig 2b). A $30 \times 25 \mathrm{~cm}$ light weight prolene mesh was kept over the above structure. Mesh was only fixed superiorly to xiphoid cartilage. Romovac suction drain of number 16 was placed. After placement of mesh, peritoneal flap was sutured with anterior sheath of left side to anterior sheath on right side which resulted in neo linea alba (Fig 2c). Another Romovac suction drain of number 16 was placed below the skin. Ulcerated and necrotic skin was removed and was approximated with skin stapler (Fig 3). The patient didn't develop abdominal compartment syndrome, surgical site infection during hospital stay. Both the drains were removed on $5^{\text {th }}$ post-operative day and patient was discharged on the same day. Patient was followed up to 6 months and there was no any evidence of recurrence of hernia.

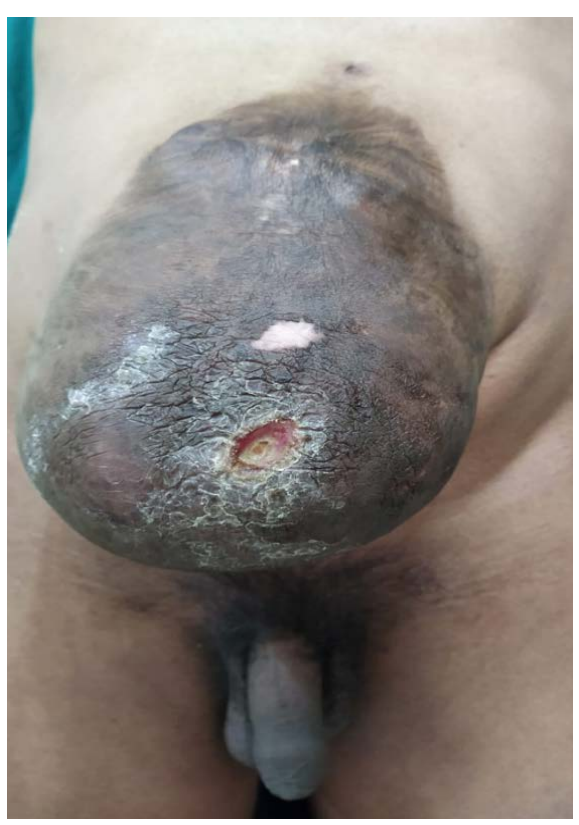

Fig 1a. Supine view of hernia

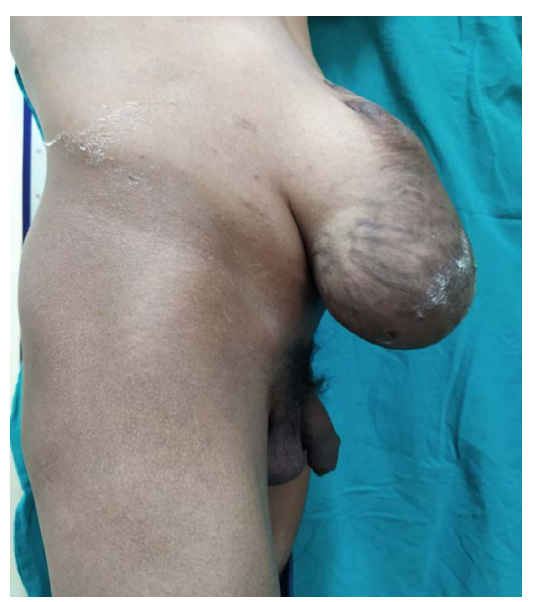

Fig 1b. Lateral view of hernia on erect position 


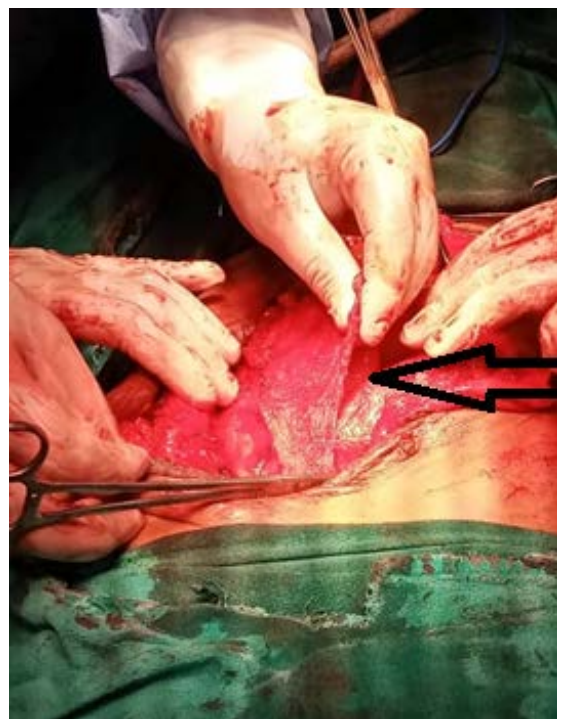

Fig 2a. Raised peritoneal flap

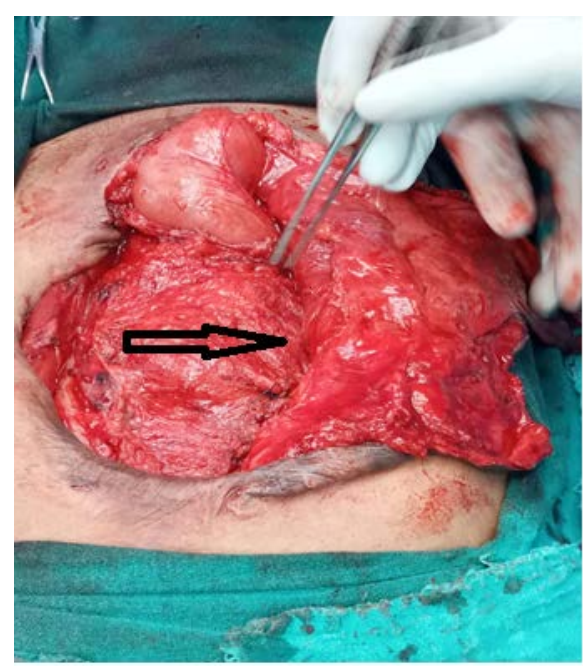

Fig 2b. Approximation of raised posterior flap with posterior sheath

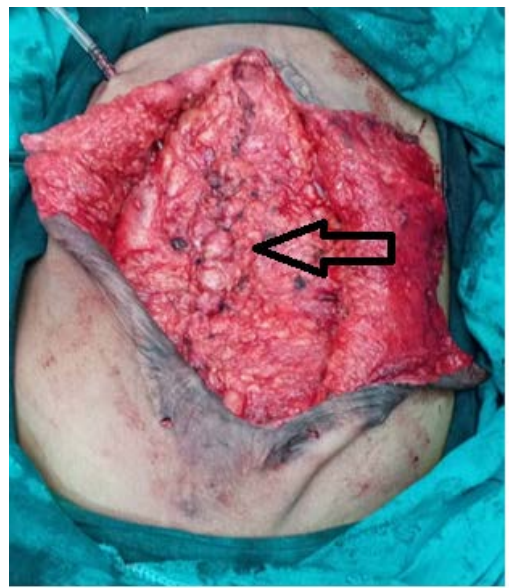

Fig 2c. New lineal alba

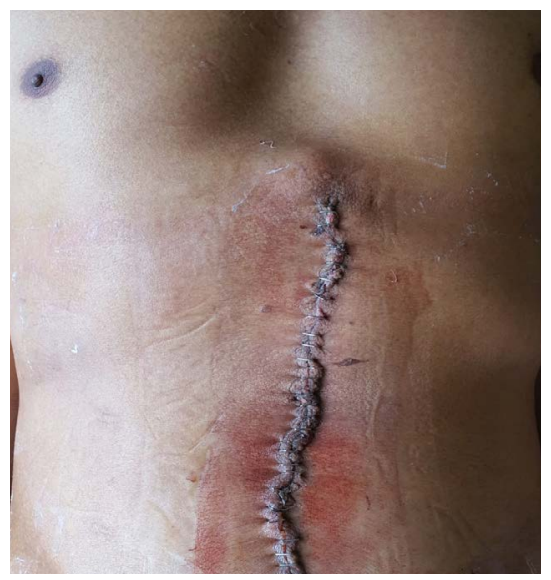

Fig 3. Final appearance of wound after approximation of healthy skin

\section{DISCUSSION}

In 2014, Malik et al. $^{5}$ described the peritoneal flap hernioplasty technique, in which flaps of the hernia sac are created by entering the hernia sac in the midline and retro muscular space through the posterior rectus fascia on one side and the anterior fascia on the other side. This technique increases abdominal domain by expanding the abdominal wall at the site of herniation without inducing weakness laterally. This is in contrast to the CSTs where tension is maximum laterally. It also reduces the need for extensive dissection, resulting in fewer complications. ${ }^{2} \mathrm{~A}$ lower incisional hernia recurrence rate for this technique as compared to retromuscular technique has been observed the study done by Petterson ${ }^{5}$ in 2019.

This technique has less evidence with troublesome bowel adhesions, erosion, fistulation, mesh infection or chronic sinus formation because the mesh is excluded from subcutaneous plane. ${ }^{4}$ As opposed to CSTs, which is only applicable to midline defects, the peritoneal flap technique may be used for reconstruction of both transverse and oblique as well as paramedian and midline incisional hernia. ${ }^{4}$

The importance of restoration of the midline rectus musculature to regain physiological function of the abdominal wall remains open to debate. A main objective for CSTs is to achieve midline fascial closure, but peritoneal flap hernioplasty doesn't attempt this. Even so, it does approximate them to a considerable degree.

It is our contention that abdominal wall function relies more on an intact circle or ring of abdominal muscle/fascia, and that it does not matter if the gap between the muscles is several centimeters wide as long as it is firm and strong, providing a firm ridge of fibrous tissue against which the 
recti and lateral obliques can pull. CSTs has the potential to affect the function of the abdominal wall from fibrosis induced by the mesh or from the extensive dissection itself. The peritoneal flap repair for midline hernias obviates these problems. There is no doubt that the triple layer neo-linea alba produced in a peritoneal flap repair provides this. Indeed, as the results from the 3-month clinical review appointment reveal, there is a high degree of patient satisfaction after the peritoneal flap repair, with very few complaints of bulge or other complaints related to the appearance or weakening of the abdominal wall. ${ }^{2}$

\section{CONCLUSION}

Owing to the favorable outcome in this case, suitability of peritoneal flap mesh hernioplasty technique in treatment of complex ventral hernia could be explored further where closure of primary defect is difficult, high risk of exposure of mesh is present and development of abdominal compartment syndrome can occur. We, therefore, recommend further studies which can establish this technique as a suitable option for management of complex ventral hernia.

\section{CONFLICT OF INTEREST}

None

\section{CONSENT FROM PATIENT}

The patient has provided consent for publication of the case.

\section{REFERENCES}

1. Slater NJ, Montgomery A, Berrevoet F, Carbonell A.M, Chang A, Franklin $M$ et al. Criteria for definition of a complex abdominal wall hernia. Hernia. 2014;18(1):717. DOI: $10.1007 / \mathrm{s} 10029-013-1168-6$

2. MF, de Beaux A, Tulloh B. Peritoneal Flap Hernioplasty for Reconstruction of Large Ventral Hernias: Long-Term Outcome in 251 Patients. World J Surg. 2019;43(9):21572163. DOI: $10.1007 / \mathrm{s} 00268-019-05011-0$

3. Malik A, MacDonald ADH, De Beaux AC, Tulloh BR. The peritoneal flap hernioplasty for repair of large ventral and incisional hernias. Hernia. 2014;18(1):39-45. DOI:10.1007/s10029-013-1086-1087

4. Farooque F, Jacombs ASW, Roussos E, Read J, Dardano A, Edye $\mathrm{M}$ et al. Preoperative abdominal muscle elongation with botulinum toxin A for complex incisional ventral hernia repair. ANZ J Surg. 2016;86(1-2):79-83. DOI:10.1111/ans.13258

5. Petersson P, Montgomery A, Petersson U. Modified Peritoneal Flap Hernioplasty Versus Retromuscular Technique for Incisional Hernia Repair: a Retrospective Cohort Study. Scand J Surg. 2019. DOI:10.1177/1457496919863943 